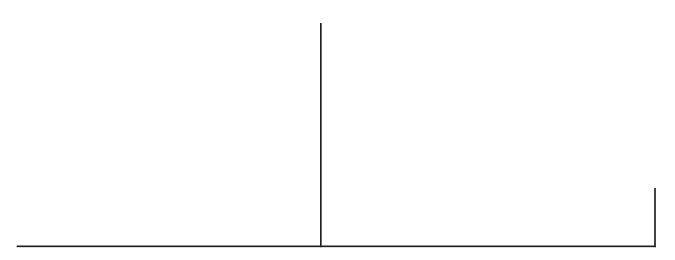

\title{
El sujeto, como singularidad, desentona en el cuadro de la clínica
}

Víctor Novoa

Entre los aspectos que en la clínica psicoanalítica continúan sin encontrar una respuesta satisfactoria están los fenómenos psicóticos presentes en el campo de las neurosis. Freud se ocupó de ellos desde el comienzo de su obra en "Estudios sobre la histeria" y los mencionó también al final de la misma en "Construcciones en el análisis", sin que al respecto planteara una resolución definitiva. Por su parte, Lacan delimita el terreno de las psicosis planteando como su mecanismo estructural a la verwerfung. Sin embargo, las neurosis y su relación con este tipo de fenómenos sigue despertando en la actualidad el interés de psicoanalistas, quienes han propuestos distintas explicaciones sin llegar a un consenso en cuanto a los mecanismos que intervienen en la producción de alucinaciones y delirios en los neuróticos, lo que indica que el tema sigue abierto a investigación. 


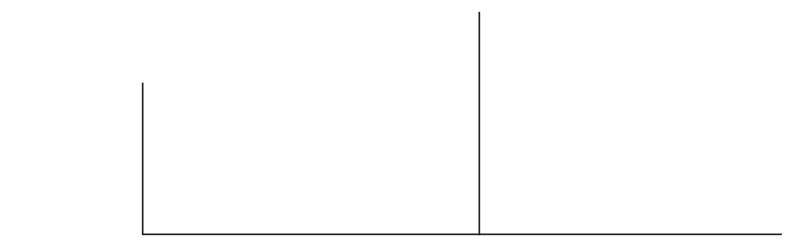

El sujeto, como singularidad, desentona en el cuadro de la clínica. Por ciertos lados, es siempre imposible ponerle nombre. El sujeto nunca está sino representado por lo que nos dice, y que puede ser situado en la estructura. Pero en cuanto al sujeto mismo, él conserva su libre albedrío. Es incluso libre como el aire, con la salvedad de que lleva una cruz a cuestas, su ser: (a). Esta cruz, evidentemente, atenúa su libertad, hasta el punto de que puede ir a ver a un psicoanalista para pedirle que lo alivie de ella.

Michel Silvestre

Hace un siglo, Freud estaba sorprendido de los resultados obtenidos con el método catártico en pacientes histéricos. Y no obstante su éxito terapéutico, reconocía los huecos en su clínica. Uno de los que más llamó su atención fue el contraste entre lo efectivo del método por un lado, y la pobreza de las explicaciones sobre las distintas formas de manifestación sintomática por el otro. Todavía en esa época el profesor de Viena era un detective del síntoma, se limitaba a reconocerlo, describirlo e investigarlo; mientras los factores que lo producían permanecían en la oscuridad. Él mismo manifiesta su inconformidad en "Estudios sobre la histeria", cuando al final del capítulo tercero afirma que está muy lejos la posibilidad de un entendimiento cabal de esta enfermedad. ${ }^{1}$

Le faltaba develar los procesos que intervienen en la formación de síntomas; tarea que logró con su teoría de los sueños, en la que formula la operación de los cuatro mecanismos que intervienen en su formación. Estos mecanismos introducen un nuevo horizonte etiológico determinado por lo que hay de irreductible, tanto en el síntoma como en el sueño. Y así, trauma y ombligo del sueño serán los conceptos fundamentales por lo que toca a las fuentes y los alcances del nuevo método lenguajero que afecta lo inasimilable sin diluirlo.

El psicoanálisis nació, entonces, al quedar establecidas las bases de una teoría del inconsciente que explica, con un soporte estructural, las distintas maneras en que los síntomas se generan.

De estos mecanismos, condensación y desplazamiento destacan como sus maestros artesanos; desde el comienzo muestran en su función la prevalencia del significante sobre la significación. Impresionante adelanto

1. S. Freud. "Estudios sobre la histeria", in Obras Completas, Buenos Aires, Amorrortu, 1978, vol. II, p. 260. 
en el campo de la linguística el de Freud. Para quien interpretar un sueño es rastrear la forma en que operan esos mecanismos, y no la búsqueda de un sentido en tanto absoluto. El proceso de deformación onírica revela la particular manera en que ellos operan, lo que directamente nos lleva a plantear la cuestión del sueño y su sentido, en la dimensión de una estructura que previamente determina al fenómeno.

Como consecuencia del invento del inconsciente, los síntomas deben ser ubicados según su relación con las deformaciones producidas por estos mecanismos en el aparato psíquico. Irán surgiendo nuevas precisiones sobre las diversas entidades clínicas, que se pondrán a prueba en cada experiencia; la delimitación estructural de la clasificación psicopatológica realizada por Freud sufrirá ajustes, en la medida en que el saber freudiano se nutre no tanto del encuentro con, sino de la búsqueda de la verdad.

En todo caso, es por la forma en que intervienen los mecanismos para producir cada síntoma, que su condición de estructura se establece. Esto significa que fenoménicamente podemos observar síntomas muy similares, sin que ello implique que surjan del mismo suelo.

Desde los inicios Freud habló de neurosis mixtas, de las múltiples formas en que podían combinarse los síntomas en una entidad clínica. Hay en sus páginas menciones a la psicosis histórica, o la histeria y la paranoia, la histeria y la melancolía, o a las formas delirantes en la neurosis obsesiva, del mismo modo que a síntomas neuróticos en las psicosis. Es cierto que se pueden establecer algunos criterios de diferenciación como son la historia, la evolución de la enfermedad, la transferencia, la demanda; es decir, con base en el modo como el sujeto responde a aquello que lo interroga.

Sin embargo, es principalmente a partir de las formaciones del inconsciente, y los efectos que éstas producen en el terreno de la subjetividad, desde donde quedará clínicamente determinada la estructura.

Los síntomas son ya efecto de un encuentro con lo real, y en el momento en que se comunican a un analista éste, desde su estratégica posición, debe transmitir eso real que es fuente de malestar para el sujeto. El hecho de relacionar el síntoma con la historia por sus puntos oscuros, es ya suficiente para producir nuevos intentos de simbolización que revelan aspectos originarios.

Hablar de estructura y darle su lugar en la clínica, es ofrecer un espacio para que los encuentros con eso indecible adquieran una dinámica y una dirección calculadas.

Si por el contrario, se dejan fuera estos elementos determinantes de las manifestaciones que aparecen en la clínica, será muy difícil distinguir y delimitar los fenómenos que inundan el campo imaginario, fenómenos que siempre encuentran respuestas tan llenas de sentido. En Freud mismo hallamos ejemplos 


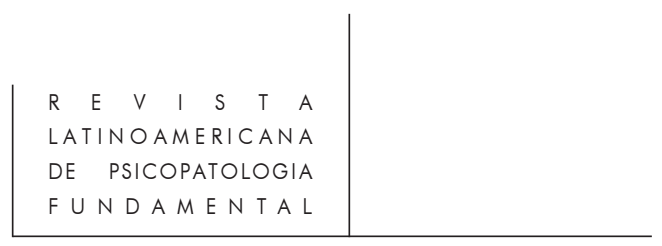

de lo que ocurre si ignoramos los factores esenciales que intervienen en la producción de los fenómenos propios de la práctica psicoanalítica; pensemos en los historiales clínicos que aparecen en sus "Estudios sobre la histeria". Cuando los revisan hay autores que resuelven algunos de los estos historiales como esquizofrénicos.

Los casos presentados en "Estudios sobre la histeria" nos ofrecen varios ejemplos de alucinación y delirio. Anna O. tenía alucinaciones de verdadero terror en las que aparecían serpientes negras, cabezas de muertos y esqueletos; además de padecer disgregación verbal. Emmy von N. veía animales y cadáveres acompañados de una rica producción delirante, así descrita por el mismo Freud:

Si hasta ahora uno acostumbra diagnosticar "histeria" en el sentido estricto, siguiendo la semejanza con los notorios casos típicos, en el de la señora Emmy von N. difícilmente se puede poner en entredicho esa designación. La prontitud para los delirios y alucinaciones, pese a una actividad espiritual en lo demás intacta, la alteración de la personalidad y de la memoria en el sonambulismo artificial, la anestesia en la extremidad dolorosa, ciertos datos de anamnesis, su neuralgia ovárica etc., no dejan duda alguna sobre la naturaleza histórica de la enfermedad contraída, o al menos de la enferma. ${ }^{2}$

Miss Lucy R. sufría de alucinaciones olfativas, a Katherina le atemorizaba una espantosa cabeza. Es decir, que de los casos presentados por Freud, únicamente uno, el de Elisabeth (quien sufría de Astacia), corresponde estrictamente a lo que entenderíamos hoy por histeria. ${ }^{3}$

Es claro que desde un principio Freud aceptó la presencia de fenómenos delirantes y alucinatorios en las neurosis. De ahí que, en sus primeros trabajos, sea frecuente hallar el término de psicosis histórica como una forma de histeria, en la que la alucinación es producto de una falla en la defensa. Y, al igual que en La interpretación de los sueños, nos encontramos con la expresión "alucinaciones histéricas".

En 1907 Freud escribía a Jung que toda histeria puede convertirse en psicosis aguda alucinatoria; no en demencia precoz, sino en "mencia" (confusión menta). Es decir, que Freud claramente reconocía la existencia de manifestaciones delirantes y alucinatorias agudas de naturaleza histórica. Mientras que si el delirio se volvía crónico, entonces ya se trataba de una estructura distinta, de tal suerte que el diagnóstico de demencia precoz era el apropiado. ${ }^{4}$

En este sentido, es necesario considerar al fenómeno como efecto de un proceso psíquico que le antecede, y de una estructura que lo determina.

2. Idem, pp. 104-105.

3. J.C. Maleval. "El escamoteo de la locura histórica", in Locuras históricas y psicosis disociativas, Buenos Aires, Paidós, 1987, pp. 232-233.

4. Idem, p. 233. 
Delirio histórico y delirio psicótico responden a procesos muy distintos; su posibilidad de análisis es mayor si se contemplan dos dimensiones del síntoma: en relación con la estructura y como metáfora. En cuanto a la primera, el síntoma aparece ligado a ese momento mítico que Freud llamó "represión originaria", donde la imposibilidad de relación sexual es marca inaugural para el sujeto, en cuanto queda instituída una falta fundante desde el universo simbólico.

En términos de Lacan, podemos referirnos a este nivel como s(A): la falta en la estructura estaría precisamente en el anudamiento primordial entre el registro de lo real y el registro de lo simbólico. A esta forma estructural del síntoma Lacan la describe como sinthome.

Y en cuanto a la segunda - la metáfora -, desde el origen la experiencia psicoanalítica muestra al síntoma íntimamente ligado a la palabra, a esa palabra no dicha en la que, en el juego del decir, se hace presente lo no dicho como insistencia que cubre de imposibilidad al síntoma mismo de llegar a significarlo todo. El trauma descubierto por Freud permanece, aunque afectado por los entrecruzamientos que se producen con el lenguaje.

La existencia de lo real en lalengua se muestra por la anticipación del significante sobre la significación, lo que se aprecia en virtud de las rupturas de sentido. Se trata de un tipo de insistencia que se efectúa, del lado de lo imaginario, en los equívocos y juegos de palabras. Que la gramática se encuentra ligada a la estructura, se observa también en "Pegan a un niño", donde los cambios gramaticales son en sí mismos soporte de la pulsión y sus transformaciones. Lo real insiste por el lado simbólico.

La ex-sistencia de lo real se genera por la instauración de la falta original y de un no-todo. El analista se enfrenta a lo indestructible del deseo por un lado, y a lo invariable de lo real por otro. Desde su lugar, exterior e inasimilable, y apuntando a la pulsión, la función analítica promueve modificaciones con respecto al estatuto que tiene el sujeto frente a lo real.

Es común en psicoanálisis hablar de las tres grandes estructuras clínicas, asociándolas con los mecanismos que intervienen de manera específica en la determinación de cada una de ellas. Así, neurosis, psicosis y perversión constituyen entidades clínicas que son resultado de lectura que Lacan hace de la obra freudiana. Y sin embargo la clínica, siempre más vasta de lo que de ella podemos decir, nos rebasa; y nos enfrenta con fenómenos que cuestionan, plantean serios interrogantes a una clasificación psicopatológica que se pretenda perfectamente clara y delimitada.

De hecho, si rastreamos el uso que Freud diera a los mecanismos represión, desestimación y desmentida - advertimos que en ninguno de ellos hay un desarrollo lineal y sí, en cambio, muchos saltos de un espacio clínico a otro. Y también los aplica a fenómenos muy diversos, lo que da por resultado un horizonte bastante confuso. 


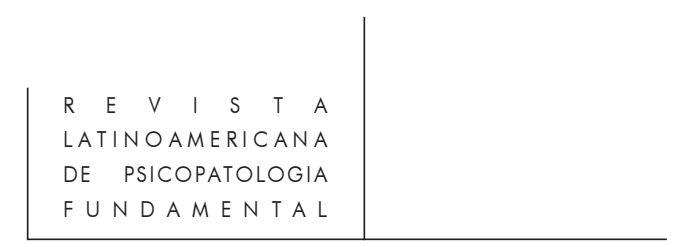

Me propongo demostrar cómo Freud utiliza desestimación y desmentida para explicar el fenómeno psicótico. El mecanismo de desestimación le sirve cuando se refiere, tanto a exigencias pulsionales, como a retoños del inconsciente o a construcciones del analista. Mientras que el mecanismo de desmentida lo aplica a recuerdos, la castración, la realidad, a lo reprimido, a la muerte.

Uno de los usos más frecuentes del mecanismo de la desestimación la planteó Freud, hace más de un siglo, en su trabajo La neuropsicosis de defensa. Ahí afirma que existe una modalidad defensiva mucho más enérgica y exitosa, consistente en que el yo desestima la representación insoportable junto con su afecto, y se comporta como si la representación jamás hubiese comparecido. Sólo que, en el momento en que lo consigue, la persona se encuentra en una psicosis que no admite otra clasificación que confusión alucinatoria. El yo se ha defendido de la representación insoportable mediante el refugio en la psicosis.

Posteriormente, cuando presenta el caso del Hombre de los lobos, Freud utiliza el término desestimación al explicar cómo, frente a la diferencia de los sexos, el sujeto desestima lo nuevo que ve. Punto privilegiado del texto freudiano, que habrá de servirle Lacan como apoyo fundamental cuando desarrolla este concepto.

Después del "Hombre de los lobos", sin embargo, la mayoría de las veces Freud no va a utilizar a la desestimación, sino a la desmentida como el mecanismo que da origen a las alucinaciones. Lo más sorprendente es que también le sirve para explicar los fenómenos alucinatorios que ocurren en neuróticos; al menos es así como aparece en "Construcciones en el análisis", uno de sus últimos trabajos. Freud aquí destaca la oposición entre verleugnung/desmentida (según el diccionario: negación, mentís, desconocimiento) y verdrängung/represión (según el diccionario: supresión, eliminación, expulsión, desalojo), pero no habla de verwerfung/desestimación (según el diccionario: rechazo, repudio, condenación, reprobación. Y para Lacan: forclusión).

$\mathrm{Al}$ respecto, en su trabajo sobre el ateísmo de Freud, Octave Mannoni reúne a desestimación y desmentida en un sólo concepto: forclusión. Al final de su desarrollo puede concluirse que el término de "repudio", tal y como él lo traduce, nos ofrece dos caras en el concepto freudiano de verleugnung: desmentida y desestimación.

Claude Raban piensa que el mecanismo de la verleugnung fue a su vez, no tanto forcluído del corpus lacaniano, sino propiamente renegado en beneficio de la forclusión y la referencia a la verwerfung ${ }^{5}$. En este sentido, el texto lacaniano

5. Cf. C. Raban. "Desestimación y forclusión. Tema conceptual", in Inventar lo real, Buenos Aires, Nueva visión, 1993, p. 228. 
conserva la huella del momento en el que Lacan eligió el concepto que utilizaría para abordar la cuestión de las psicosis. Gesto de rechazo o renegación, según Raban, de la verleugnung.

En el Seminario sobre La Psicosis, el 15 de febrero de 1956, Lacan menciona la objeción que le hacen por emplear el concepto de verwerfung, ¿por qué no verleugnung? - lo interrogan sus discípulos. Lacan responde:

Si hay cosas de las que el paciente nada quiere saber, refiriéndose al "Hombre de los lobos", incluso en el sentido de la represión esto supone otro mecanismo, y como la palabra verwerfung aparece en conexión directa con esta frase (...) echo mano de ella. El término no me importa especialmente, me importa lo que quiere decir, y creo que Freud quiso decir eso. Los que más objeciones me hacen, proponen ir a buscar en tal o cual otro texto de Freud algo que no sería verwerfung, sino por ejemplo la verleugnung (...). Ténganme un poco de confianza en lo tocante a este trabajo de los sentidos, si elijo verwerfung para hacer comprender es el fruto de una maduración. Mi trabajo me condujo a ello. Reciban al menos por un tiempo mi miel tal y como se las ofrezco, e intentemos hacer algo con ella. ${ }^{6}$

Es una imposición lógica de Lacan delimitar el campo, a partir de proponer como operación fundante de la psicosis el rechazo de un significante primordial: la forclusión del Nombre-del-Padre.

Desde esta propuesta, la lectura freudiana se ordena por los ejes trazados en el Seminario de La Psicosis, y se opta por fragmentos que definitivamente dirigen el llamado "retorno a Freud".

La desestimación será "una modalidad defensiva más enérgica y exitosa" que la represión, según la definió Freud en 1894. Este mecanismo supone cierto trámite de energía que erosiona aquello sobre lo cual recae, a diferencia de la represión en la que lo "desalojado" conserva toda su virulencia.

En 1964, en su respuesta al comentario de Jean Hyppolite, Lacan menciona que para designar ese "no querer saber nada" en el sentido de la represión, Freud emplea el término verwerfung. Entonces no se trata de una represión, pues la represión no puede distinguirse del retorno de lo reprimido, aquello de lo que no puede hablar el sujeto, pero lo grita por todos los poros de su ser. Cito a Lacan:

Su efecto es una abolición simbólica, cercena la castración y con ello no puede decirse que fuese propiamente formulado juicio alguno sobre su existencia, pero fue exactamente como si nunca hubiese existido. ${ }^{7}$

6. J. Lacan. "Del rechazo de un significante primordial", in El seminario de Jacques Lacan. Libro 3. Las psicosis 1955-1956, Barcelona, Paidós 1985, pp. 216-217.

7. J. Lacan. "Respuesta al comentario de Jean Hyppolite", in Escritos II, México, Siglo XXI, 1975, p. 147. 


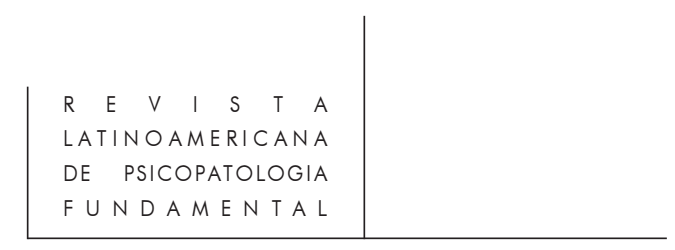

La diferencia va a ubicarse en el nódulo del concepto de verneinung negación. Se trata, dice Lacan, de un proceso ubicado en uno de los tiempos de la dialéctica de la negación bajo el nombre de verwerfung, y se opone a la bejahung (afirmación) primaria. Constituye lo que es expulsado, es decir, que la verwerfung se encuentra en lo que ha quedado fuera del campo simbólico, de la bejahung primordial en la que toma su raíz el juicio de atribución, y en donde se encuentran las condiciones para esos primeros encuentros con la introyección simbólica, cuando la afirmación inaugural queda ligada al discurso del inconsciente. Aquello que ha cercenado, el sujeto no podrá encontrarlo en su historia, ni tampoco podrá historizarlo: "Lo que no ha llegado a la luz de lo simbólico aparece en lo real".

El otro tiempo, la ausstossung, expulsión fuera del sujeto, constituye lo real en cuanto se trata del dominio de aquello que subsiste fuera de la simbolización; de ahí que la castración cercenada quede sustraída de toda posibilidad de la palabra.

Es decir, que en un primer momento se produce la expulsión primaria, cuyo efecto es la delimitación de lo real como exterior al sujeto. La representación se constituye después, por la reproducción de aquella primera percepción; además de que recibe derecho de existencia, el objeto puede reencontrarse.

El sentimiento de extrañeza se produce, para el "Hombre de los lobos", cuando él se encuentra con el símbolo que cercenó en el origen de su bejahung. De esta experiencia no queda relación alguna con lo imaginario, se presenta por eso como congelada en un tiempo indefinido, del que Lacan dice que es un "estado", no sólo de inmovilidad en la que se hunde, "sino en una especie de embudo temporal, de donde regresa sin haber podido contar las vueltas de su descenso y de su ascenso, y sin que su retorno a la superficie del tiempo común hubiese respondido de su esfuerzo". ${ }^{8}$

Según Lacan, la forclusión del Nombre-del-Padre era el mecanismo determinante en la alucinación del "Hombre de los lobos". Pero no obstante el voto de confianza que les pidió, al menos dos de sus discípulos manifestaron sus reservas.

Safouan señaló que la forclusión que se advierte en este caso no es del mismo orden que la del presidente Schreber. Distingue la forclusión como mecanismo de defensa, tal y como se presenta en el "Hombre de los lobos", de la forclusión en cuanto defecto primordial de lo simbólico.

Mannoni, por su parte, estima que la forclusión del Nombre-del-Padre sería un caso particular, el más grave; pero reconoce que habría también otras formas de forclusión.

8. Idem, p. 151. 
En la presentación de las obras completas de Freud para la Editorial Amorrortu, al trabajar el concepto de desmentida, Etcheverry anota que verwen significa arrojar, de ahí que verwerfen en su primera acepción quiere decir "desechar". Desestimar entonces, de acuerdo con el uso que da Freud a este concepto, equivale a un "no, no es así, eso no tiene la importancia que pretende".

Y sobre el caso del "Hombre de los lobos" en particular, Etcheverry aclara que desestimación es una de las categorías nucleares de este análisis.

Cuando el paciente, en su infancia, tuvo la evidencia visual de la diferencia entre los sexos, se comportó como lo hacen todos los niños frente a un esclarecimiento indeseado. Movido por la angustia de castración (tenía 4 años), Er verwarf das Neue, desestimó (o mejor aún: rechazó, según la traducción literal) eso nuevo que veía y se atuvo a su vieja creencia. Er Entschied sich, se decidió en favor de la teoría de la cloaca y en contra de la existencia de la vagina. ${ }^{9}$

Es importante ver la relación que existe entre desestimar en un primer momento, y decidirse en un segundo momento. Lo nuevo, la diferencia sexual es rechazada por el niño, es decir, se produce un "no ha lugar"; y aunque conserva la vieja teoría de la cloaca, lo nuevo desestimado produce efectos sobre las formaciones del inconsciente. El esfuerzo de desalojo que ejerce sobre el sueños de los lobos es constante. Cumplida esta función, la desestimación ya no influye en la decisión del problema sexual del paciente, y es aquí donde Freud aclara que la represión es algo distinto. El significado más inmediato de desestimación, es que no quiso saber nada de eso, siguiendo el camino de la represión. ${ }^{10}$

No obstante la relación entre desmentida y represión es muy estrecha, tanto, que parecieran confundirse - el mecanismo de la desmentida se ejerce sobre elementos de lo reprimido, excluyendo cualquier forma de retorno que tienda a la simbolización.

En este sentido, el "no ha lugar" de la desestimación no parece ser tan definitivo como lo trabaja Lacan. Ya que, en La interpretación de los sueños encontramos diferentes usos y dimensiones del concepto. Un deseo pudo haberse excitado durante el día, sin que se realizara a causa de las condiciones objetivas, y entonces, admitido pero no tramitado, se queda pendiente para la noche, en este caso el deseo se halla en el preconsciente. Otro caso es el de un deseo que emerge de día y se topa con una desestimación, tampoco se tramita pero queda sofocado, es decir, fue esforzado hacia atrás del preconsciente hasta el

9. S. Freud. "Sobre la versión castellana", in Obras Completas, Buenos Aires, Amorrortu, 1978 , p. 69.

10. Idem, pp. 69-71. 


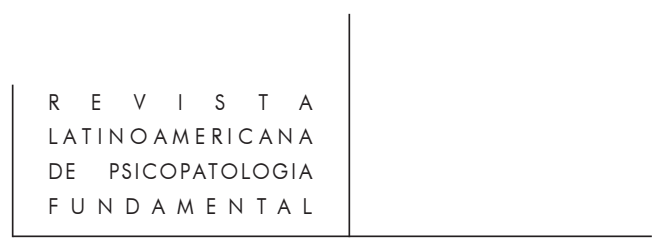

inconsciente. $\mathrm{Y}$ un tercer caso lo constituyen aquellos deseos que no tienen relación alguna con la vida diurna, y que sólo se ponen en movimiento durante la noche desde lo ya sofocado. Estos deseos no pueden salir del inconsciente.

Lo desestimado puede entonces quedar en el preconsciente y ser susceptible de volverse consciente, o bien quedar sofocado en el inconsciente; ese "no ha lugar" derivado de un juicio parece tocar más a lo reprimido por el lado de lo simbólico. Con el ejemplo que nos ofrece en el caso del "Hombre de los lobos", Lacan aclara el concepto de forclusión del Nombre-del-Padre. Y a su vez va más allá de lo elaborado por la teoría freudiana sobre la desestimación, delimitando retroactivamente el terreno de la clínica. La pregunta es: ¿No hay muchos fenómenos clínicos que, forzosamente, quedarían incluídos o excluídos si se respeta rígidamente esta postura?

Hay un deslizamiento, del primer al último Freud, entre desestimación y desmentida. La desmentida tiene que ver con el examen o prueba de realidad; algo objetivo se desmiente, y entonces se abre una brecha por la cual la alucinación irrumpe. En "La pérdida de la realidad en la neurosis y la psicosis" 1924 -, Freud afirma que la neurosis intenta tramitar el conflicto, o bien se reprime la exigencia pulsional en cuestión desavaloriza la alteración objetiva y se desvaloriza así la alteración objetiva, o bien se tramita la reacción psicótica desmintiendo la realidad objetiva. ${ }^{11}$

Un año después, en "Algunas consecuencias psíquicas de la diferencia anatómica entre los sexos", Freud señala que la desmentida, que no es ni rara ni peligrosa en la infancia, en el adulto podría llevar a una psicosis.

Y en "El fetichismo" - 1927 - afirma que el mecanismo de la desmentida puede dar origen a una neurosis obsesiva, o bien a una psicosis; la diferencia sería que en ésta última "el yo se deja arrastrar por el ello a desprenderse de una parte de la realidad". Y establece la diferencia entre represión y desmentida. La primera, dice, se aplica como defensa contra las demandas pulsionales internas, mientras que la desmentida es defensa contra los reclamos de la realidad.

Aun cuando la desmentida se refiere a algún fragmento de la realidad que tiene que ver con la diferencia anatómica entre los sexos, es decir que remite directamente a la castración, es cierto que puede ampliar sus efectos sobre otros elementos de la realidad. Y es aquí donde es preciso delimitar el campo de la desestimación. Porque en este mismo texto afirma Freud que el yo del fetichista desmiente un fragmento sustancial de la realidad: el desagradable hecho de la castración en la mujer.

11. S. Freud. "La pérdida de la realidad en la neurosis y en la psicosis", in Obras completas, Buenos Aires, Amorrortu, 1978, vol. XIX, p. 195. 
Y esto quiere decir que coexisten en él las dos corrientes: la acorde con el deseo y la corriente acorde con la realidad. Esta última, la acorde con la realidad, faltaría en el caso de la psicosis.

Encontramos pues que el mecanismo de la desmentida interviene de manera determinante en la perversión, en la neurosis y en la psicosis.

También hay algunos pasajes en la obra de Lacan, en los que de modo rotundo vincula el mecanismo de la desmentida con lo real. En noviembre de 1975, en las conclusiones de las jornadas de la Escuela freudiana dice, por ejemplo, que la relación de la desmentida con lo real es indudable, pero que no obstante también mantiene su relación con lo imaginario, como algo persistente bajo la forma de espejismo en el caso de la perversión para el neurótico.

Y Maleval distigue así al delirio psicótico del neurótico. El primero, dice, aparecería como un retorno de lo real que se muestra disociado y explotado del lenguaje, en la imposibilidad del sujeto de hacer uso de la palabra, de metáfora. Mientras que en el caso de la neurosis, la formación delirante se encontraría ubicada en un desarrollo eminentemente imaginario, cargado de significación y con posibilidad de hacer metáfora.

También en el "Seminario de la lógica del fantasma" - el 15 de febrero de 1967 - cuando habla sobre la repetición y el acto, Lacan trata de manera más directa la relación entre la desmentida y lo real. Y se refiere a este mecanismo en tanto interviene en aquello que de la estructura, es decir, que del sujeto frente a lo real, se modifica en la incidencia del acto. Cito a Lacan:

Es preciso considerar que la verleugnung, término al cual, apoyándose en Freud, querían referirse los efectos que he reservado a la verwerfung - se distingue de esto: lo que es del orden de la verleugnung es siempre lo que tiene que ver con la ambigüedad que resulta de los efectos del acto como tal.

Se trata de un encuentro directo y de una transformación subjetiva, en donde Lacan enfatiza la función que la desmentida desempeña, para designar el reconocimiento de los efectos del acto sobre el sujeto. Hacia el final de este mismo Seminario, afirma que es imposible decidir si un acto puede ser imitado "en tanto que no se sabe, en cada uno de los niveles en que se le podía distinguir, cuál es el efecto del acto. Ahora bien, es este laberinto - propio al reconocimiento para un sujeto de efectos que no puede reconocer, puesto que, como sujeto, está totalmente transformado por su acto - que desgina, en cualquier parte donde el término es justamente empleado, la rúbrica de la verleugnung".

El vínculo con lo real y la afectación de lo real por surcos tan cercanos no conduce sino a confundirnos; o al menos a preguntarnos, como lo hace Raban, si el concepto de la verleugnung hace borde con la forclusión, o si llega a interactuar con ella. Incluso podría pensarse que la verleugnung es un elemento 


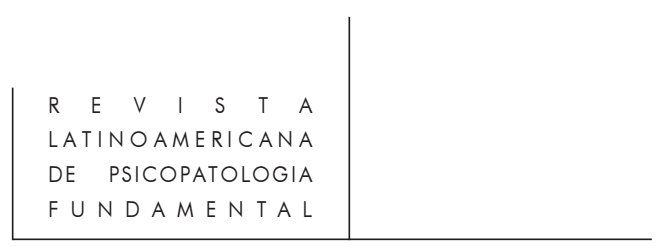

regulador, que interviene en los procesos originales mediante los cuales el sujeto construye su realidad.

\section{Resumos}

Entre os aspectos que na clínica psicanalítica ainda não encontraram uma resposta satisfatória, estão os fenômenos psicóticos presentes no campo das neuroses. Desde o início de sua obra, em "Estudos sobre a histeria", Freud se ocupou deles mencionando-os também em "Construções na análise", sem que chegasse a uma resolução definitiva.

Lacan, por sua vez, delimita o terreno das psicoses propondo como seu mecanismo estrutural, a verwerfung. Sem dúvida, as neuroses e sua relação com este tipo de fenômeno ainda desperta, atualmente, o interesse dos psicanalistas que já propuseram várias explicações, sem, contudo, chegarem a um consenso quanto aos mecanismos que intervêm na produção de alucinações e delírios nos neuróticos, indicando que a investigação do tema continua aberta.

Parmi les aspects de la clinique psychanalytique qui n'ont toujours pas reçu de réponse satisfaisante, nous retrouvons les phénomènes psychotiques présents dans le champ des névroses. Dès le début de son cuvre, dans "Etudes sur l'hystérie", Freud s'est penché sur eux, et les a également mentionnés dans "Constructions dans l'analyse", sans toutefois arriver les résoudre définitivement.

Lacan, à son tour, cerne le champ des psychoses en proposant la verwefung comme leur mécanisme structurel. Les névroses et leur relation avec ce type de phénomènes éveillent donc encore, de nos jours, l'intérêt des psychanalystes qui ont déjà proposé plusieurs explications sur les mécanismes qui interviennent dans la production d'hallucinations et de délire chez les névrosés, ce qui montre que l'investigation reste en suspens.

Among the aspects of clinical psychoanalysis lacking a satisfactory answer are the psychotic phenomena present in the field of neuroses. Since the beginning of his works, in "Studies on Hysteria", Freud worried about them, mentioning them again in "Construction in Analysis" without coming to a definitive solution.

Lacan, as for him, delineates the field of psychosis proposing the verwefung as their structural mechanism. Beyond doubt, neuroses and their relationship to this phenomenon still awaken, nowadays, the interest of psychoanalysts who have already proposed various explanations, though they have not yet come to a consensus as for the mechanisms interfering in the production of hallucinations and delirium in neurotic people, thus revealing that the investigation on this theme is still open. 